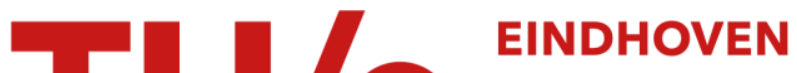 UNIVERSITY OF TECHNOLOGY
}

\section{Intermittency and scaling laws for wall bounded turbulence}

\section{Citation for published version (APA):}

Benzi, R., Amati, G., Casciola, C. M., Toschi, F., \& Piva, R. (1999). Intermittency and scaling laws for wall bounded turbulence. Physics of Fluids, 11(6), 1284-1286. https://doi.org/10.1063/1.869993

DOI:

10.1063/1.869993

Document status and date:

Published: 01/01/1999

\section{Document Version:}

Publisher's PDF, also known as Version of Record (includes final page, issue and volume numbers)

\section{Please check the document version of this publication:}

- A submitted manuscript is the version of the article upon submission and before peer-review. There can be important differences between the submitted version and the official published version of record. People interested in the research are advised to contact the author for the final version of the publication, or visit the $\mathrm{DOI}$ to the publisher's website.

- The final author version and the galley proof are versions of the publication after peer review.

- The final published version features the final layout of the paper including the volume, issue and page numbers.

Link to publication

\section{General rights}

Copyright and moral rights for the publications made accessible in the public portal are retained by the authors and/or other copyright owners and it is a condition of accessing publications that users recognise and abide by the legal requirements associated with these rights.

- Users may download and print one copy of any publication from the public portal for the purpose of private study or research.

- You may not further distribute the material or use it for any profit-making activity or commercial gain

- You may freely distribute the URL identifying the publication in the public portal.

If the publication is distributed under the terms of Article $25 f a$ of the Dutch Copyright Act, indicated by the "Taverne" license above, please follow below link for the End User Agreement:

www.tue.nl/taverne

Take down policy

If you believe that this document breaches copyright please contact us at:

openaccess@tue.nl

providing details and we will investigate your claim. 


\title{
Intermittency and scaling laws for wall bounded turbulence
}

\author{
R. Benzia) \\ Autoritá Informatica Pubblica Amministrazione, via Solferino 15, 00185 Roma, Italy and Istituto Nazionale \\ di Fisica della Materia, Unità di Tor Vergata, Italy \\ G. Amati \\ Consorzio Applicazioni Supercalcolo Per Universitá e Ricerca, p.le A. Moro 5, 00185 Roma, Italy \\ C. M. Casciola \\ Dip. Mecc. Aeron., Università di Roma, “La Sapienza," via Eudossiana 18, 00184 Roma, Italy \\ F. Toschi \\ Dip. di Fisica, Università di Pisa, Piazza Torricelli 2, 56126 Pisa, Italy and Istituto Nazionale di Fisica \\ della Materia, Unità di Tor Vergata, Italy \\ R. Piva \\ Dip. Mecc. Aeron., Università di Roma, “La Sapienza," via Eudossiana 18, 00184, Roma, Italy
}

(Received 1 December 1998; accepted 4 March 1999)

\begin{abstract}
Well defined scaling laws clearly appear in wall bounded turbulence, very close to the wall, where a distinct violation of the refined Kolmogorov similarity hypothesis (RKSH) occurs together with the simultaneous persistence of scaling laws. A new form of RKSH for the wall region is here proposed in terms of the structure functions of order two which, in physical terms, confirms the prevailing role of the momentum transfer towards the wall in the near wall dynamics. (c) 1999 American Institute of Physics. [S1070-6631(99)03706-X]
\end{abstract}

The intermittent behavior of velocity increments in the inertial range of fully developed turbulence has been a subject of renewed interest during the years, starting from the objection that Landau raised to Kolmogorov theory of 1941 (K41). Since then, any theory of the inertial range cannot avoid considering the effect of intermittent dissipation of energy on the inertial scales of motion. Under this respect, the Kolmogorov-Obukhov refined similarity hypothesis (RKSH), certainly the most credited, ${ }^{1}$ leads to a probability distribution function of longitudinal velocity increments, $\delta V$ $=V(x+r)-V(x)$, characterized by the scaling

$$
\left\langle\delta V^{p}\right\rangle \propto\left\langle\epsilon_{r}^{p / 3}\right\rangle r^{p / 3},
$$

where $\epsilon_{r}^{q}$ denotes the $q$ th moment of the energy dissipation rate $\epsilon$ spatially averaged over a volume of characteristic dimension $r$ and the brackets indicate ensemble averaging. Taking into account the scaling properties of the dissipation field,

$$
\left\langle\epsilon_{r}^{q}\right\rangle \propto r^{\tau(q)},
$$

Eq. (1) implies that the velocity structure function of order $p$ is expressed as a power law of the separation with exponent

$$
\zeta_{p}=\tau(p / 3)+p / 3 .
$$

Here, the anomalous correction, $\tau(p / 3)$, to the K41-exponent accounts for the intermittency of the velocity increments in the inertial range of homogeneous and isotropic turbulence.

A substantial extension of the range of scales where similarity is observed has recently been achieved ${ }^{2}$ by assuming, as a basic quantity, the third order structure function instead of the separation $r$,

$$
\left\langle\delta V^{p}\right\rangle \propto\left(\left\langle\epsilon_{r}^{p / 3}\right\rangle /\langle\epsilon\rangle^{p / 3}\right)\left\langle\delta V^{3}\right\rangle^{p / 3},
$$

as suggested by the Kolmogorov equation. ${ }^{1}$ A direct consequence of Eq. (4) is the existence of an extended selfsimilarity (ESS) of the generic structure function of order $p$ in terms of the third order moment with exponent $\zeta_{p}$.

In the present letter we discuss the issue of intermittency and scaling laws in wall bounded turbulence. We suggest a new theoretical interpretation of the intermittent behavior near the wall which violates Eq. (4). We support our findings by a comparison against a direct numerical simulation of a turbulent channel flow, ${ }^{3}$ and references therein, performed on a $256 \times 128^{2}$ grid, up to $t \simeq 10^{3} h / U_{0}$, where $h$ is the channel half-width and $U_{0}$ the mean centerline velocity. The simulation is based on a Lattice Boltzmann method ${ }^{4}$ implemented on a massively parallel computer. We remark that the dimensions of the channel we adopt in the calculations $(4 h \times 2 h$ $\times 2 h$ ) are smaller than required for the correlation functions to vanish at large separations. ${ }^{5}$ Such a choice has been dictated by the need of a very long simulation to achieve accurate statistics and fully resolved dissipation range in all directions. Despite the above limitations, the present numerical results are encouraging even though further numerical and experimental investigations should be performed to confirm the theoretical predictions proposed here. From our numerical simulation we have evidence, as shown in Fig. 1, that the intermittency increases moving from the bulk of the fluid towards the wall. ${ }^{3}$ In principle, one may attempt to describe this behavior in the framework of RKSH, in its generalized form (4). Hence the larger intermittency (smaller $\zeta_{p}$ ) would be provided by an increase of intermittent fluctuations of $\epsilon_{r}$ (larger values of $|\tau(p)|$ ). In such conditions, the anomaly of 

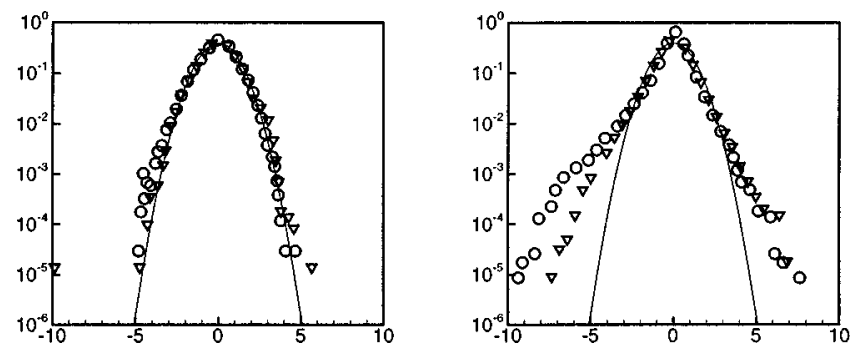

FIG. 1. Pdf of the velocity increments for different values of the separation (left $r^{+}=160$, right $r^{+}=18$ ) at two distances from the wall; $y^{+}=151$, near the center of the channel, triangles, and $y^{+}=31$, in the wall region of the flow, circles. Data from DNS of a turbulent channel flow with $\mathrm{Re}_{*}=160$. Wall units are used throughout the paper.

the scaling exponents would strongly depend on the local flow properties, losing, thus, any trait of universality. To investigate the self-consistency of this approach, in Fig. 2 we plot on a logarithmic scale the structure function of order six vs $\left\langle\epsilon_{r}^{2}\right\rangle\left\langle\delta V^{3}\right\rangle^{2}$. On the basis of the assumed validity of (4), the plot should result in a straight line of slope $s=1$, independent of the distance from the wall. This behavior actually emerges near the center of the channel while in the wall region a quite clear, though small, violation is manifested. Specifically, for $y^{+}=31$ two different scaling laws appear. The one, characterized by slope $s=1$, trivially pertains to the dissipative range. The other, with slope $s=0.88$, which does not satisfy (4), shows a first clear example of failure of RKSH.

The previous discussion may suggest a relationship between the increase of intermittency, observed in the near wall region, and the simultaneous breaking of the RKSH. To this regard, it seems interesting to investigate the possible existence of a new form of RKSH valid in the near wall region. In fact RKSH, somehow suggested by the well known " $4 / 5$ ", Kolmogorov equation (see Frisch $^{1}$ ), tells us, in physical terms, that the "energy flux" in the inertial range, represented by the term $\delta V^{3}$, fluctuates with a probability distribution which is the same of $\epsilon_{r}$. However, in the case of strong shear, we should expect that a new term, proportional to $\partial_{y} U \delta V^{2}$, enters the estimate of the energy flux at scale $r$.

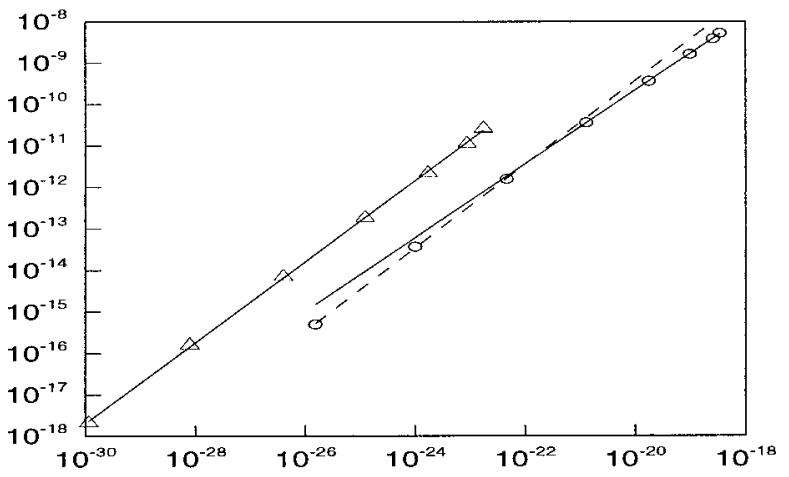

FIG. 2. $\left\langle\delta V^{6}\right\rangle$ vs $\left\langle\epsilon_{r}^{2}\right\rangle\left\langle\delta V^{3}\right\rangle^{2}$ for two different wall normal distances. Bulk region $\left(y^{+}=151\right)$, data (triangles) and their fit in the region $r^{+} \in[20,320]$ (solid line with slope 0.99). Wall region $\left(y^{+}=31\right)$, data (circles) and their fits in the two regions $r^{+} \in[1,20]$ and $r^{+} \in[20,320]$, dotted line with slope 0.99 and solid line with slope 0.88 , respectively.

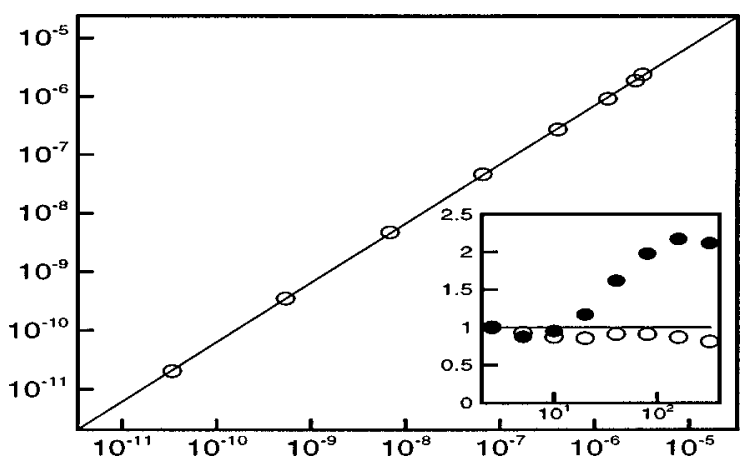

FIG. 3. Check of consistency for Eq. 5 at $y^{+}=31,\left\langle\delta V^{4}\right\rangle$ vs $\left\langle\epsilon_{r}^{2}\right\rangle\left\langle\delta V^{2}\right\rangle^{2}$. The solid line (slope 1.01) gives the fit in the whole range. In the insert, open circles, compensated plot for Eq. (5), $\left\langle\epsilon_{r}^{2}\right\rangle\left\langle\delta V^{2}\right\rangle^{2} /\left\langle\delta V^{4}\right\rangle$ vs $r^{+}$; filled circles, corresponding plot for Eq. (4), $\left\langle\epsilon_{r}^{2}\right\rangle\left\langle\delta V^{3}\right\rangle^{2} /\left\langle\delta V^{6}\right\rangle$ vs $r^{+}$.

Such a new term, indeed, appears in the analysis performed for homogeneous shear flows (see for instance Hinze ${ }^{6}$ ). If this term becomes dominant, as it may occur for a very large shear, one is led to assume that the fluctuations of the energy flux in the inertial range are proportional to $\delta V^{2}$, i.e., $\epsilon_{r}$ $\propto A(r) \delta V^{2}$, with $A(r)$ a nonfluctuating function of $r$. Hence, we may expect that a new form of the RKSH should hold which, in its generalized form,

$$
\left\langle\delta V^{p}\right\rangle \propto\left(\left\langle\epsilon_{r}^{p / 2}\right\rangle /\langle\epsilon\rangle^{p / 2}\right)\left\langle\delta V^{2}\right\rangle^{p / 2},
$$

is given in terms of the structure function of order two. In the spirit of the extended self-similarity, we assume the new form of RKSH to be valid in the region very close to the wall, where the shear is certainly prevailing.

In order to support this set of assumptions, we show in Fig. 3 a $\log -\log$ plot of Eq. (5) for $p=4$ at $y^{+}=31$. In the insert, we show for the same plane the compensated plot of both (5) and (4) for $p=4$ and $p=6$, respectively. It follows a quite clear agreement of Eq. (5) with the numerical data. In principle, the function $A(r)$ might be evaluated theoretically starting from the Kolmogorov equation for anisotropic shear flow (e.g., see Ref. 7).

The increased intermittency of the velocity fluctuations near the wall may be estimated by considering how the flatness $F(r)$ grows with $r \rightarrow 0$, with

$$
F(r)=\left\langle\delta V^{4}(r)\right\rangle /\left\langle\delta V^{2}(r)\right\rangle^{2} .
$$

By combining the definition (6) with (4) and (5), respectively, we obtain the following expressions in terms of $\epsilon_{r}$ :

$$
F_{b}=\left\langle\epsilon_{r}^{4 / 3}\right\rangle /\left\langle\epsilon_{r}^{2 / 3}\right\rangle^{2}, \quad F_{w}=\left\langle\epsilon_{r}^{4 / 2}\right\rangle /\left\langle\epsilon_{r}^{2 / 2}\right\rangle^{2},
$$

which are suitable for the bulk and the near-wall region, respectively. As we see from Fig. 4 , both $F_{b}$ and $F_{w}$ manifest a significant growth for $r \rightarrow 0$, indicating intermittent behavior, if we exclude the smallest separations falling into the dissipative range. Clearly, $F_{w}$ grows faster than $F_{b}$. This result is consistent with the corresponding analysis performed directly in terms of structure functions of velocity by means of Eq. (6) and provides a further evidence of the validity of (5) near the wall. In fact, the application of $F_{b}$ near the wall does not catch the increase of intermittency of the velocity fluctuations (see Fig. 4). On the other hand, the dif- 


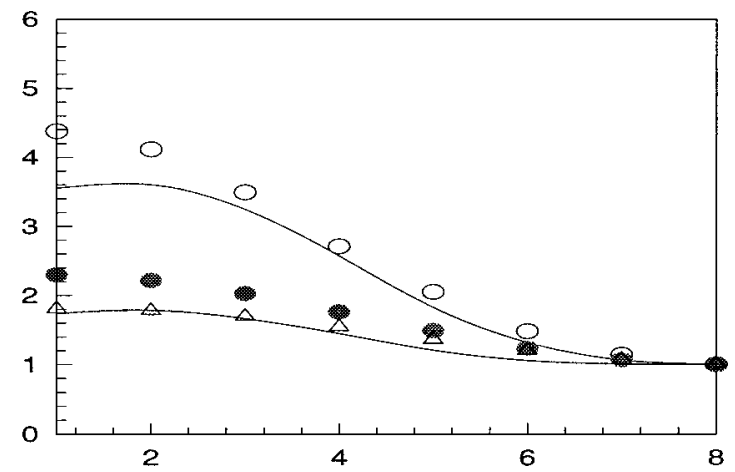

FIG. 4. Flatness, $F=\left\langle\delta V^{4}\right\rangle\left\langle\delta V^{2}\right\rangle^{2}$ vs $\log _{2}\left(r^{+} / D x^{+}\right), D x^{+}=2.5$, at $y^{+}=151$ (open triangles) and $y^{+}=31$ (open circles), as evaluated by Eqs. (7), using $F_{b}$ and $F_{w}$, respectively. For comparison, filled circles, $F_{b}$ applied at $y^{+}$ $=31$. Correspondingly, the solid lines give the flatness as evaluated directly in terms of velocity.

ferences in the statistical properties of the dissipation between the bulk and the near wall region are too small to account for the increase of intermittency of the velocity increments near the wall. This is indirectly confirmed by the observed direct scaling (ESS) of the structure functions with $\left\langle\delta V^{3}\right\rangle$, which implies, starting from Eq. (5),

$$
\hat{\tau}(p / 2)=\hat{\zeta}_{p}-\hat{\zeta}_{2} p / 2,
$$

where a hat has been introduced here to denote the scaling exponents with respect to $\left\langle\delta V^{3}\right\rangle$. This distinction was not necessary in the bulk region, where $\tau \equiv \hat{\tau}$. By using expression (8) near the wall and Eq. (3) in the bulk region we obtain that the "intermittency correction" $\hat{\tau}(q)$ results to be essentially independent of the distance from the wall, Fig. 5. Hence the observed increase of intermittency of the velocity increments seems to be associated more to the structure of the RKSH rather than to the intermittency of dissipation. These theoretical findings are in good agreement with experimental results in a flat plate boundary layer obtained recently by Ciliberto and co-workers (private communication) and, independently, by us. We like here to emphasize that, to observe the new RKSH, we selected on purpose the plane closest to the wall where the scaling exponents can still be computed. On the opposite, in the bulk region, the original RKSH holds. At intermediate planes we expect the scaling exponents to emerge from a complex blending of these two basic behaviors, leading to a continuous variation with the distance from the wall. ${ }^{3}$

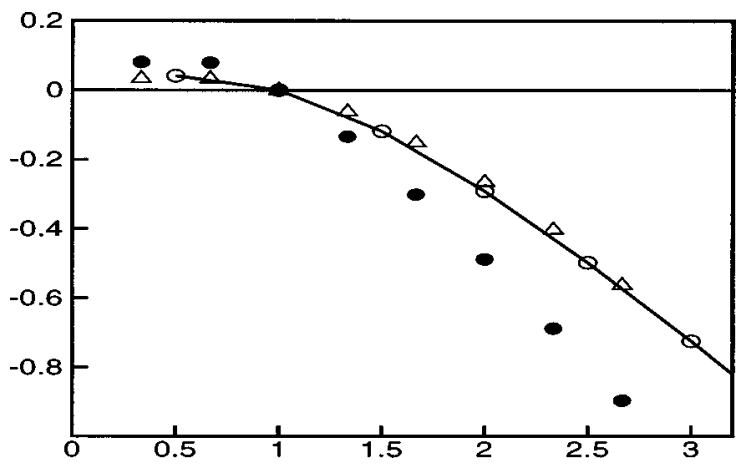

FIG. 5. The anomalous correction $\hat{\tau}(q)$ as computed by the new scaling law for the wall region $\left(y^{+}=31\right)$, Eq. (5), (open circles) compared with that issuing from RKSH at both $y^{+}=151$ (triangles) and $y^{+}=31$ (filled circles).

In conclusion, we have a strong evidence that a significant failure of the RKSH occurs in the near wall turbulence in correspondence with the simultaneous appearance of scaling laws. The new form of the RKSH we propose in this letter for the wall region is expressed in terms of the structure function of order two, instead of the structure function of order three as in the original form. This may be seen as a statistical representation of the physical features of the near wall region, which is controlled more by the mechanism of momentum transfer rather than by the classical energy cascade.

\section{ACKNOWLEDGMENTS}

We acknowledge very useful discussions with $L$. Biferale, S. Succi, and S. Ciliberto.

a) On leave of absence from Dip. di Fisica, Università di Roma, “Tor Vergata," Italy.

${ }^{1} \mathrm{U}$. Frisch, Turbulence: The legacy of A. N. Kolmogorov (Cambridge University Press, Cambridge, 1995).

${ }^{2}$ R. Benzi, L. Biferale, S. Ciliberto, M. V. Struglia, and R. Tripiccione, “Generalized scaling in fully developed turbulence,'” Physica D 96, 162 (1996).

${ }^{3}$ G. Amati, S. Succi, R. Piva, and F. Toschi, "Scaling exponents in turbulent channel flow," Advances in Turbulence VII (Kluer Academic, Dordrecht, 1998), p. 159.

${ }^{4}$ S. Chen, and G. D. Doolen, "Lattice Boltzmann method for fluid flows," Annu. Rev. Fluid Mech. 30, 329 (1998).

${ }^{5}$ J. Kim, P. Moin, and R. Moser, "Turbulence statistics in a fully developed channel flow at low Reynolds number," J. Fluid Mech. 177, 133 (1987).

${ }^{6}$ J. O. Hinze, Turbulence (McGraw-Hill, New York, 1959).

${ }^{7}$ I. Arad, B. Dhruva, S. Kurien, V. S. L'vov, I. Procaccia, and K. R. Sreenivasan, "The extraction of anisotropic contributions in turbulent flows,’' Phys. Rev. Lett. 81, 5330 (1998). 